\title{
História, epidemiologia e controle da malária na região de Campinas, Estado de São Paulo, Brasil, 1980 a 2000
}

\author{
History, epidemiology and control of malaria in Campinas \\ region, São Paulo State, Brazil, 1980 to 2000
}

Maria José Chinelatto Pinheiro Alves $\quad{ }^{1}$, Renata Caporalle Mayo ${ }^{1}$ e Maria Rita Donalisio ${ }^{2}$

RESUMO

Estudo retrospectivo descritivo dos 3.314 casos de malária notificados na área de abrangência da Superintendência de Controle de Endemias, Campinas (88 municípios, 5.366.081 habitantes), no período de 1980 a 2000. Foram considerados elementos da bistória da expansão da malária na região. Houve queda dos casos diagnosticados mesmo em periodos de recrudescimento da malária na Amazônia. Predominaram homens (83\%), em idadeprodutiva (20 a 49 anos), vindos principalmente de Rondônia, Pará e Mato Grosso; 59\% foram diagnosticados nos 3 primeiros dias dos sintomas. Considerou-se o possível impacto positivo de campanhas educativas endereçadas às populações de risco e aos profissionais de saúde na região. Em áreas não endêmicas, a assistência oportuna ao paciente com malária, a vigilância epidemiológica/entomológica e a ações educativas podem diminuir a gravidade dos casos e impedem o estabelecimento de focos de transmissão.

Palavras-chaves : Malária. Anopheles. Programa de controle. Vigilância epidemiológica. Endemia.

\section{ABSTRACT}

It is a retrospective descriptive study about 3314 notified cases of malaria in Superintendência de Controle de Endemias, Campinas, an area that covers 88 cities and 5.366.081 inhabitants, during 80's and 90's. The region data were compared to national statistics looking at disease bistory and control program impact in endemic areas. It was observed a decrease in notified cases in Campinas region, while over the same period incidence in Amazonia was increasing. The highest prevalence was in men (83\%) between 20 and 49 years old, mainly from Rondônia, Pará and Mato Grosso State; 59\% were diagnosed in three first days of symptoms. It was suggested the impact of educational campaigns addressed to risk population and health professionals in the Campinas region. In no endemic areas, early diagnostic and treatment are a great challenge because it may extend patient access and proper heath care decreasing lethality and impeding local transmission. It can also facilitate integration among vector control, epidemiologic surveillance and attendance to patients.

Key-words : Malaria. Anopheles. Control program. Epidemiologic surveillance. Endemic disease.

Desde o reconhecimento das febres palúdicas, tem sido assinalada a importância das condições ambientais aliadas às transformações sócio-econômicas na transmissão de doença ${ }^{4}$.

No Estado de São Paulo, o desmatamento e o amplo processo migratório permitiram a instalação, consolidação e aumento da doença ${ }^{8}$. 0 declínio das atividades de extração mineral levou algumas áreas do Estado, em fins do século XVIII, particularmente a região de Campinas, a se voltar para a cultura da cana de açúcar, mobilizando o capital gerado na fase da mineração. A elaboração industrial do açúcar exigia o largo consumo de um combustível, a lenha, que naturalmente tinha que ser retirada das matas que circundavam os engenhos e canaviais na região. A extensão dessa exploração agrícola determinou, por necessidade, a diminuição das áreas de vegetação florestal ${ }^{17}$.

Alguns anos após a Independência, nova exigência da devastação foi imposta às áreas pertencentes à região de Campinas, com a introdução de novo produto agrícola: o café. Mas esta lavoura não se limitava às terras já conhecidas e ocupadas pela cana, avançou

1. Superintendência de Controle de Endemias Regional, Campinas, SP. 2. Epidemiologia do Departamento de Medicina Preventiva e Social da Faculdade de Ciências Médicas da Universidade de Campinas, Campinas, SP.

Endereço para correspondência: Dra. Maria José Chinelatto Pinheiro Alves. SUCEN/Regional-5. R. São Carlos 546, Vila Industrial, 13035-420 Campinas, SP.

e-mail: sr05@sucen.sp.gov.br

Recebido para publicação em 4/4/2003

Aceito em 3/12/2003 
para as regiões mais elevadas dos terrenos cristalinos à leste. E 0 círculo foi se alargando com o aumento das áreas agrícolas, crescimento das populações rurais e urbanas ${ }^{17}$.

A intervenção direta do governo privilegiando a economia cafeeira fez com que a fronteira agrícola sofresse maior deslocamento em busca de novas áreas para a expansão da cultura do café. A relação entre o processo de ocupação e as doenças do sertão era uma realidade concreta. A malária era então o grande flagelo para quem se aventurasse para o interior, principalmente para a região dos grandes rios, onde a temperatura é elevada e permitia o desenvolvimento da cultura do café13.

Nos primeiros anos do século XX, São Paulo ingressou na fase mais dinâmica do processo de ocupação. É nessa fase que a fronteira agrícola sofreu o maior deslocamento em busca de novas áreas para a expansão do café ${ }^{817}$.

Na região de Campinas, no período de 1910 a 1920 os relatos jornalísticos apontavam a epidemia da malária em vilas próximas ${ }^{14}$. Registrou-se na região a presença do $A$. ( $N$.) darlingi com grande expressão e dispersão. 0 anofelino habitava os vales dos rios Mogi Guaçú, Piracicaba, Tietê, Jaguari Mirim, de onde se espalhavam, subindo os afluentes desses grandes rios ${ }^{15}$.

Em 1932, no Serviço Sanitário do Estado, foi criada a Seção de Estudos e Profilaxia do impaludismo, transformada em 1933 na Inspetoria de Profilaxia do Impaludismo, semente da atual Superintendência de Controle de Endemias (SUCEN) ${ }^{813}$. Crescia então neste período, a incidência da malária no Estado de São Paulo. No ano de 1936, foi assinalada a existência da doença em 149 dos 258 municípios existentes ${ }^{15}$.

Nas décadas de 30 a 50, a malária foi uma doença freqüente em quase todas as áreas do Estado. Nesse período, verificou-se uma grande migração da zona rural para a urbana, a qual passou a conter $51,2 \%$ da população. Este fato, aliado à redução do desbravamento territorial e ao início da industrialização, gerou possibilidades de regressão da doença ${ }^{8}{ }^{13}$. 0 novo modelo de desenvolvimento privilegiou a indústria em detrimento da agricultura, particularmente após a segunda metade da década de $50^{13}$.

Na região de Campinas, atualmente constata-se que, das matas primitivas nada mais resta. Foram devastadas quer pela necessidade de utilização de seus componentes (madeira, lenha), quer pela necessidade de utilização de seus espaços para o plantio. As condições ecológicas para a transmissão da malária foram se tornando menos propícias.

A erradicação da malária no Estado de São Paulo/região de Campinas. Na IX Assembléia Mundial da Saúde, reunida em Genebra, em 1956, aprovou resolução na qual solicita “... a todos os governos que procurem alcançar a erradicação da malária antes que se concretize o perigo de resistência aos inseticidas pelas espécies vetoras..."

Em 1958 foi elaborado para o estado de São Paulo o Plano para a Erradicação da Malária, sendo demarcada a área malárica ${ }^{15}$. Neste período, as pesquisas entomológicas realizadas não apontavam a presença do A.(N.) darlingi na regiãa $0^{101516}$.

$\mathrm{Na}$ década de 60, a Campanha de Erradicação modificou radicalmente o comportamento epidemiológico da malária no
Estado, derrubando as taxas de incidência da doença ${ }^{15}$. Esta ocorrência foi possível em várias regiões do estado, devido aos pressupostos para a erradicação, ou seja, a transmissão principalmente domiciliar, a não existência de reservatórios animais e a disponibilidade de um inseticida de efeito residual eficaz, o DDT. Estes requisitos não estavam presentes em outras regiões como a Norte e a Centro-Oeste ${ }^{1218}$.

A intensificação pela busca de casos e a implantação da rede de notificantes ${ }^{8}$, elevaram de 3.525 casos de malária em 1959 para 10.179 em 1960. A partir daí observa-se queda acentuada da incidência da doença no estado. Ficou demonstrado que as ações de erradicação, padronizadas e transformadas em rotina de trabalho, aliadas à organização técnica e operacional e aos recursos financeiros, materiais e humanos recebidos, lograram êxito nesta estratégia campanhista, produzindo o impacto esperado sobre a incidência da doença ${ }^{81316}$.

Em 1965, foi interrompido o uso do DDT na região de Campinas, Sorocaba, Ribeirão Preto e parte do litoral do estado. No período de 65 a 68, não houve registros de malária autóctone nas regiões de Campinas e Ribeirão Preto. Houve porém, focos em Sorocaba e no litoral, indicando assim que o impacto da Campanha de Erradicação não se deu de forma homogênea ${ }^{816}$.

Na segunda metade da década de 70 e na década de 80, com 0 processo de ocupação da Amazônia e a abertura de rodovias nas regiões Norte e Centro-Oeste, observou-se um considerável aumento no número de casos de malária importada na região de Campinas $^{131920}$. Apesar da situação estar sob controle, focos ocasionais da doença foram registrados em algumas regiões do Estado de São Paulo, como a de Presidente Prudente onde, no município de Panorama, no ano de 1984 foram diagnosticados 10 casos por $P$. falciparum ${ }^{5}$.

Esse trabalho se propõe a analisar a evolução dos casos de malária diagnosticados na região de Campinas, considerando-se alguns elementos da história da malária na região. Além disso, procura apontar estratégias que têm sido encaminhadas para 0 enfrentamento da doença em regiões não endêmicas.

\section{MATERIAL E MÉTODOS}

Estudo retrospectivo descritivo de todos os casos de malária diagnosticados e notificados pela rede de laboratórios da Superintendência de Controle de Endemias, Regional Campinas, no período de 1980 a 2000. Esta área está localizada a noroeste do estado de São Paulo, composta por 88 municípios, com população em torno de 5.366.081 habitantes (IBGE 2000). Trata-se de área não endêmica, porém grande receptora de casos importados.

Foram estudadas 3.114 fichas de notificação epidemiológica e examinadas as seguintes variáveis: data de diagnóstico da doença, sexo, faixa etária(na ocasião do diagnóstico), local provável de infecção, modo de transmissão, intervalo entre sintomas/tratamento, espécie de plasmódio envolvida e evolução da doença.

As fontes de dados foram os relatórios técnicos e as notificações dos casos da SUCEN, registrados a partir de exames microscópicos 
obtidos nas lâminas de sangue, originárias dos indivíduos com suspeita de malária.

A escolha desse período para análise deve-se à intensa ocorrência de casos na região Amazônica, grande exportadora de pacientes infectados para a região de estudo, nas décadas de 80 e 90.

\section{RESUL TADOS}

No período de 1980 a 2000 foram investigados e confirmados 3.114 casos de malária na região de Campinas (Tabela 1), sendo que 2.239 (71,9\%) foram devido à infecção por Plasmodium vivax, enquanto $760(24,4 \%)$ pelo Plasmodium falciparum e 115 (3,7\%) pela infecção mista destes parasitos (Figura 1).

Tabela 1 - Distribuição dos casos de malária segundo a classificação epidemiológica. Região de Campinas, SP, 1980 a 2000.

\begin{tabular}{|c|c|c|c|c|c|c|c|c|c|c|}
\hline \multirow[b]{2}{*}{ Ano } & \multirow[b]{2}{*}{ Casos $\left(n^{0}\right)$} & \multirow[b]{2}{*}{ Variação (\%) } & \multicolumn{2}{|c|}{ Importado } & \multicolumn{2}{|c|}{ Autóctone } & \multicolumn{2}{|c|}{ Induzido } & \multicolumn{2}{|c|}{ Recaída } \\
\hline & & & $\mathrm{n}^{0}$ & $\%$ & $\mathrm{n}^{0}$ & $\%$ & $\mathrm{n}^{\circ}$ & $\%$ & $n^{0}$ & $\%$ \\
\hline 1980 & 116 & - & 116 & 100,0 & - & - & - & - & - & - \\
\hline 1981 & 136 & 17,20 & 134 & 98,6 & 1 & 0,7 & 1 & 0,7 & 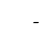 & - \\
\hline 1982 & 125 & $-8,00$ & 125 & 100,0 & - & - & - & - & - & - \\
\hline 1983 & 195 & 56,00 & 193 & 99,0 & 1 & 0,5 & 1 & 0,5 & - & - \\
\hline 1984 & 212 & 8,70 & 204 & 96,2 & - & - & 1 & 0,4 & 7 & 3,3 \\
\hline 1985 & 221 & 4,24 & 213 & 96,3 & - & - & - & - & 8 & 3,7 \\
\hline 1986 & 285 & 28,90 & 271 & 95,0 & - & - & - & - & 14 & 5,0 \\
\hline 1987 & 308 & 8,07 & 291 & 94,4 & 1 & 0,3 & - & - & 16 & 5,1 \\
\hline 1988 & 235 & $-23,70$ & 219 & 93,2 & - & - & - & - & 16 & 6,8 \\
\hline 1989 & 256 & 8,93 & 242 & 94,6 & - & 0 & 1 & 0,4 & 13 & 5,0 \\
\hline 1990 & 201 & $-21,40$ & 190 & 94,6 & - & - & 1 & 0,5 & 10 & 5,0 \\
\hline 1991 & 154 & $-23,30$ & 142 & 92,2 & 1 & 0,7 & 2 & 1,4 & 9 & 5,9 \\
\hline 1992 & 141 & $-8,44$ & 119 & 84,4 & - & - & - & - & 22 & 15,7 \\
\hline 1993 & 83 & $-41,13$ & 76 & 91,6 & 1 & 1,2 & 2 & 2,4 & 4 & ,8 \\
\hline 1994 & 113 & 36,14 & 109 & 96,4 & - & - & - & - & 4 & 3,6 \\
\hline 1995 & 83 & $-26,55$ & 76 & 91,6 & - & - & - & - & 7 & 8,4 \\
\hline 1996 & 59 & $-28,90$ & 52 & 88,1 & - & - & - & - & 7 & 11,9 \\
\hline 1997 & 43 & $-27,12$ & 39 & 90,7 & 1 & 2,3 & - & - & 3 & 7,0 \\
\hline 1998 & 57 & 32,56 & 51 & 89,4 & 1 & 1,8 & - & - & 5 & 8,8 \\
\hline 1999 & 48 & $-15,79$ & 37 & 77,0 & 2 & 4,1 & - & - & 9 & 18,8 \\
\hline 2000 & 43 & $-10,42$ & 35 & 81,3 & 1 & 2,3 & - & - & 7 & 16,2 \\
\hline Total & 3.114 & & 2.934 & 94,2 & 10 & 0,3 & 9 & 0,3 & 161 & 5,1 \\
\hline
\end{tabular}

Os casos de malária importada de regiões endêmicas representam 94,2\% do total investigado no período. A procedência destes casos aponta os estados de Rondônia, Pará e Mato Grosso como os principais estados da área endêmica, envolvidos na transmissão. Os casos importados de outros países, principalmente da África, contribuíram com percentual em torno de 4\% do total dos casos importados.

As Figuras 1 e 2 mostram a distribuição temporal dos casos da região com tendência decrescente, a partir de meados da década de 80. A tendência dos diagnósticos e malária importada na região de Campinas acompanha a registrada na região amazônica até meados dos anos $80^{10}{ }^{15}$ (Figura 3). Após este período as notificações da malária se estabilizam na região endêmica e voltam a crescer a partir de 1998, enquanto os casos diagnosticados continuam a diminuir na região de Campinas (Figuras 2 e 3).
A malária incidiu em ambos os sexos, com destacado predomínio do masculino (83\%) sobre o feminino (17\%) e em todas as faixas etárias, registrando-se maior freqüência em homens entre 20 a 49 anos, portanto em idade produtiva.

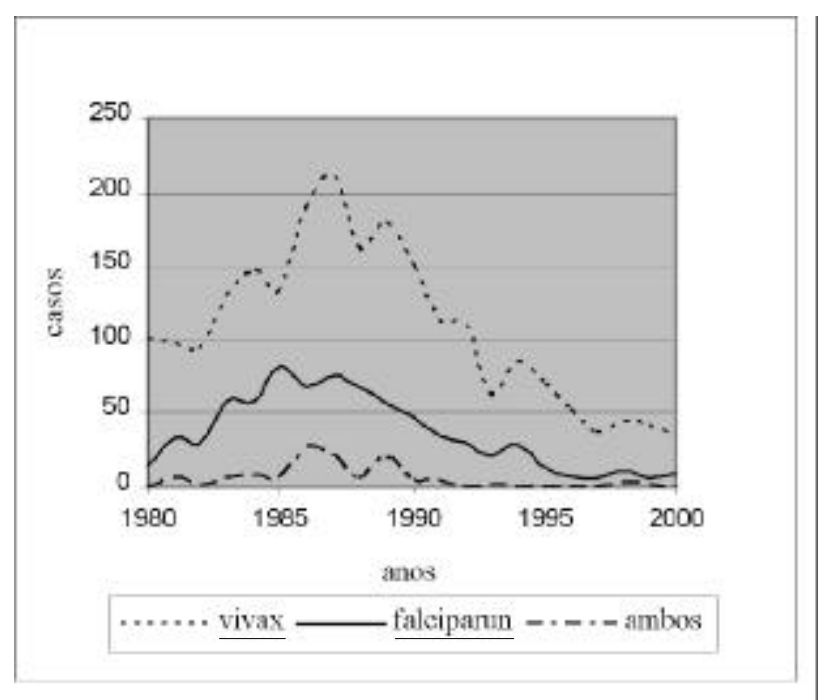

Figura 1 - Casos investigados de malária segundo a espécie de Plasmódio. Região de Campinas SP, 1980 a 2000.

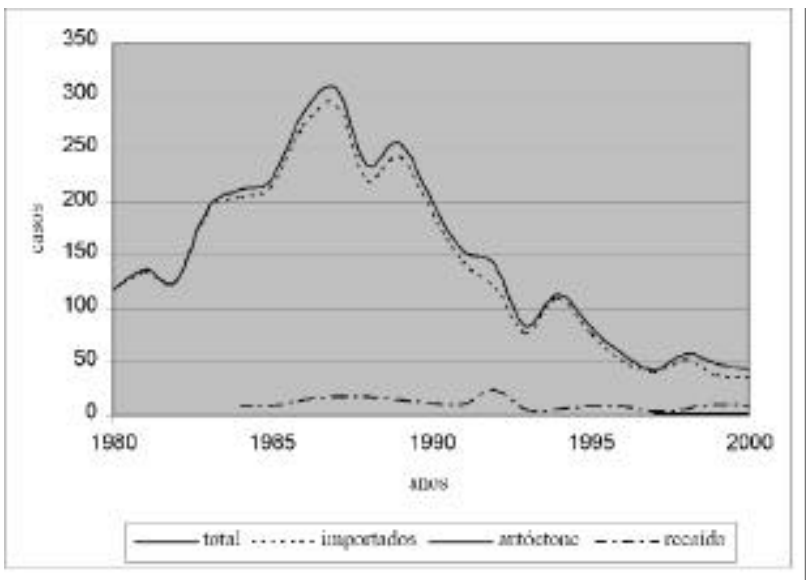

Figura 2 - Casos de malária diagnosticados na região de Campinas SP, 1980 a 2000

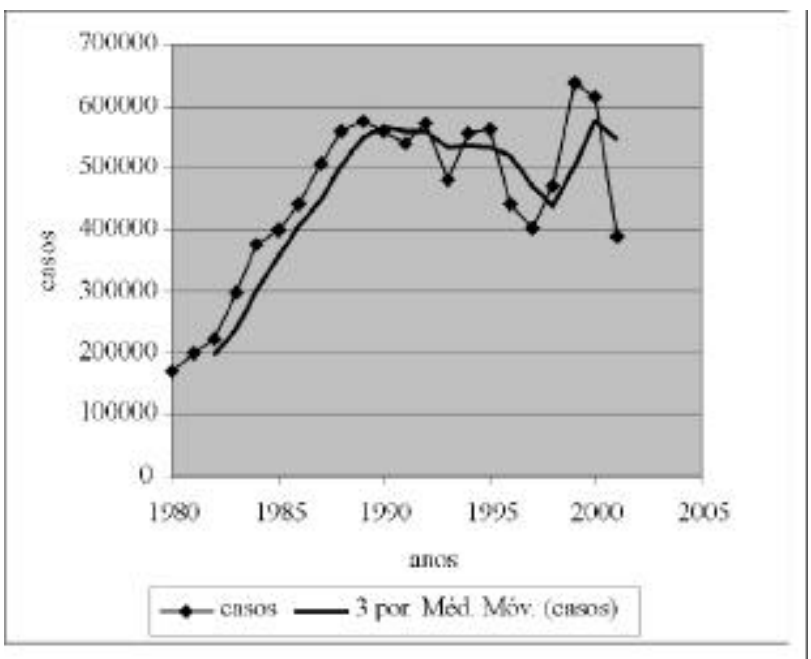

Figura 3 - Tendência temporal dos casos de malária notificados, média móvel. Brasil 1980 a 2001. 
As ocupações apontadas com maior freqüência nos locais de transmissão foram a agropecuária $(21,3 \%)$, a extração mineral (16\%) e atividade de transporte (13,2\%). 0 lazer foi responsável por 16,8\% do deslocamento de indivíduos para a área de transmissão.

No período avaliado foram diagnosticados 9 casos autóctones de outras regiões do estado, e 1 caso autóctone de infecção por P. falciparum da região de Campinas, representando 0,3\% do total dos casos confirmados. Este último cuja transmissão provável se deu no município de Mogi-Guaçú, recebeu esta classificação em virtude da ausência de dados epidemiológicos e entomológicos. Foram encontrados somente exemplares de Anopheles (N.) albitarsis, vetor secundário da malária em local próximo à residência do doente.

Em apenas 1 doente foi detectada a presença do P. falciparum, nos demais casos as infecções ocorreram em razão da presença do P. vivax. Quanto ao local de transmissão, os 9 casos ocorreram em municípios do Vale do Ribeira ou Vale do Paraíba. Essas regiões cobertas pela Mata Atlântica são consideradas por alguns autores como área endêmica para malária ${ }^{318}$ reconhecidamente com a presença de Anopheles (K.) cruzi e (K.) belattor. No registro anual de casos predominaram as infecções por $P$. vivax, com baixa parasitemia.

A procura de diagnóstico e tratamento ocorreu nos 3 primeiros dias dos sintomas da doença em $59 \%$ dos pacientes. A procura entre $04^{\circ}$ e $09^{\circ}$ dia foi referida por $32 \%$. Os indivíduos com sintomatologia malárica que procuraram o atendimento do serviço após o $10^{\circ} \mathrm{dia}$ representaram 9\% do total dos casos diagnosticados.

A infecção da malária sem a participação do vetor esteve presente em $9(0,3 \%)$ casos do total diagnosticado. Destes, 5 pacientes contraíram a doença por transfusão sangüínea, infecção por $P$. vivax e, 3 pelo uso compartilhado de seringas e agulhas, 2 deles portadores de $P$. vivax e 1 de $P$. falciparum. $09^{\circ}$ caso foi classificado como malária congênita por apresentar precocidade de sintomas, ausência de punções venosas ou transfusões sangüíneas no paciente e ausência de transmissão vetorial no município de permanência do mesmo.

Foram registrados 6 óbitos, todos portadores de malária por P. falciparum. 0 coeficiente de letalidade da malária por todos os tipos de parasita foi de 0,2 por 100 doentes na região, enquanto somente para os portadores de malária por $P$. falciparum foi de 0,79 por 100 doentes.

\section{DISCUSSÃO}

No estado de São Paulo e na região de Campinas, nas décadas de 40 e 50 predominava a malária autóctone ${ }^{813}$, a partir da década de 60 a doença passou a ser quase que exclusivamente importada da Região Amazônica, principalmente nos estados de Rondônia, Mato Grosso e Pará1 ${ }^{13}$. Nas décadas de 70 e 80, quando a ocupação de terras e o aproveitamento dos recursos naturais passaram a atrair um grande contingente populacional para a Amazônia Legal, ocorreu o aumento expressivo da malária, refletindo o aumento do diagnóstico de casos importados na região de Campinas.
Quanto à representatividade dos registros de casos da região, ressaltase no período a freqüente subnotificação da malária, como também deficiências no registro de óbitos, devido ao precário preenchimento dos atestados, ao sub-registro e à morte domiciliar " sem assistência médica", particularmente em décadas mais distantes ${ }^{14}$.

A situação epidemiológica da malária na região de Campinas, a partir da década de 80 é semelhante à dos países desenvolvidos. A doença deixa de ser uma ameaça para o conjunto da população e passa a ser um problema que afeta grupos restritos de indivíduos em função de suas atividades profissionais ou de seus comportamentos $^{12389}$. Na região de Campinas, o quadro epidemiológico apresenta a mesma tendência, exigindo das instituições de controle de vetores e vigilância epidemiológica adaptações de suas estratégias de prevenção e controle da doença. A região de Campinas passou a ser a $2^{a}$ Região Administrativa do Estado a contribuir com casos de malária importada.

0 perfil dos pacientes diagnosticados no Estado de São Paulo e na região indica tratar-se de homens, em idade produtiva e com ocupações relacionadas à exploração da Amazônia Legal. Estes dados têm subsidiado ações de educação em saúde endereçadas a grupos de maior risco e a profissionais de saúde, no sentido de facilitar o diagnóstico e tratamento ou encaminhamento oportunos, minimizando o risco de estabelecimento de focos de transmissão ${ }^{139192021}$. Embora o número de casos de malária induzida não tenha sido expressiva no período, novos grupos de risco passaram a ser incorporados nos programas de controle 26711 .

A precocidade da procura por serviços após os sintomas (59\% dos pacientes nos 3 primeiros dias após o início dos sintomas) pode estar indicando o conhecimento adequado de riscos por parte dos pacientes infectados, além da disponibilidade de serviços. Estes dados reforçam a importância de se manter em Campinas, a vigilância epidemiológica e ações de educação em saúde a grupos mais vulneráveis à infecção $0^{32021}$.

Em áreas não endêmicas, a assistência médica, a vigilância epidemiológica/entomológica oportunas e ações de educação em saúde são as estratégias prioritárias para diminuir a gravidade dos casos e impedir o estabelecimento de focos de transmissão. Atualmente o grande desafio do Programa de Controle da Malária para o Estado de São Paulo é a descentralização de atendimento aos suspeitos, procurando ampliar 0 acesso do paciente e garantir a assistência integral, vinculando o diagnóstico e o tratamento da malária ao Sistema Único de Saúde.

\section{REFERÊNCIAS BIBLIOGRÁFICAS}

1. Alves MJCP, Barata LCB, Almeida MCRR, Gutierrez EB, Wanderley D MV, Andrade JCR. Aspectos sócio-econômicos dos indivíduos com malária importada na região metropolitana de São Paulo, Brasil - Caracterização da população e conhecimento sobre a doença. Revista de Saúde Pública de São Paulo 24:253258,1990 .

2. Alves MJCP, Lima VLC, Rangel 0. Malária congênita no município de Leme - SP. Jornal de Pediatria 71:163-165, 1995.

3. Alves MJCP, Rangel 0, Souza SSAL. Malária na região de Campinas, São Paulo, Brasil, 1980 a 1994. Revista da Sociedade Brasileira de Medicina Tropical 33: 53$60,2000$.

4. Agudello SF. Saúde e Imperialismo: a ação antimalárica na América Latina Centro de Investigaciones Médicas - Universidade de Antioquia, Medellin, 1981. 
5. Andrade JCR, Anjos CFD, Wanderley DMV, Alves MJCP, Campos PC. Foco de malária no Estado de São Paulo, Brasil. Revista de Saúde pública, 20:323-26,1986.

6. Barata LCB, Andriguetti MTM. Cortas MC, Meneguetti LC. - Nota sobre surto de malária em usuários de drogas injetáveis. Revista Saúde Pública. São Paulo, 24 : $321-22,1990$

7. Barata LCB, Andriguetti MTM, Matos MR. Surto de malária induzida entre usuários de drogas injetáveis. Revista de Saúde Pública 27: 9-14, 1993.

8. Barata RB. Malária e seu controle. Saúde em Debate - Editora Hucitec Ltda - São Paulo, 1998.

9. Capuano DM, Gomes SA, Polidorio SRB. Malária na região de Ribeirão Preto, São Paulo, Brasil - 1984 a 1993. Revista Instituto Adolfo Lutz 56: 1-8, 1996.

10. Correia RC. Informes sobre o programa de erradicação da malária no Estado de São Paulo. Revista de Saúde, São Paulo, 3: 93-104, 1969.

11. Loo SS, Andrade JCR, Condino MLF, Alves MJCP, Semeghini MG, Galvão EC Malária em usuários de drogas de administração endovenosa associada à soropositividade para HIV. Revista de Saúde Pública, São Paulo, 25: 17-22, 1991.

12. Loyola CCP. Silva CJM. Tauil PL. Controle da Malária no Brasil 1965 a 2001. Revista Panamericana de Saúde Pública 11:235-244, 2002.

13. Matos RM. A malária em São Paulo. Epidemiologia e história. Editora Hucitec Ltda, São Paulo, 2000.
14. Secretaria de Estado da Saúde - Superintendência de Controle de Endemias - 50 anos de Luta, São Paulo, 1984.

15. Serviço de Profilaxia da Malária do Governo do Estado de São Paulo. Plano para a Erradicação da Malária no Estado de São Paulo - Brasil (documento mimeografado), 1958

16. Serviço de Profilaxia da Malária do Governo do Estado de São Paulo. Relatório de Avaliação Epidemiológica do Programa de Erradicação da Malária no Estado de São Paulo - Região de Campinas (documento mimeografado), 1971.

17. Silva LJ. A evolução da Doença de Chagas no Estado de São Paulo. Saúde em Debate, Editora Hucitec Ltda. São Paulo, 1999.

18. Silveira AC, Rezende DF. Avaliação da estratégia global de controle integrado da malária no Brasil. Organização Panamericana de Saúde(documento mimeografado), 2001.

19. Wanderley DMV, Andrade JCR, Alves MJCP, Mattos MR, Gurgel SM, Igreja RP. Malária no Estado de São Paulo: Avaliação dos aspectos da vigilância epidemiológica. Cadernos de Saúde Pública 5: 296-304, 1989.

20. Wanderley DMV, Andrade JCR, Meneguethi LC, Alves MJCP. Malária no Estado de São Paulo - Brasil, 1980 a 1983. Revista Saúde Pública, São Paulo 19: 28-36, 1985.

21. Wanderley DMV, Silva RA, Andrade JC. Aspectos epidemiológicos da malária no Estado de São Paulo, Brasil - 1983 a 1992. Revista de Saúde Pública 28: 192-197, 1994 